\title{
Absence of mutations at SERPINI1 gene in a cohort of patients with Cerebral Cavernous Malformations
}

\author{
Concetta Scimone, ${ }^{1,2}$ Rosalia D’Angelo, ${ }^{1,2}$ Simona Alibrandi, ${ }^{1,3}$ Fabiana Nicita, ${ }^{1,2}$ Luigi Donato, ${ }^{1,2}$ \\ Antonina Sidoti ${ }^{1,2}$ \\ ${ }^{1}$ Department of Biomedical and Dental Science and of Morphological and Functional Images, University of Messina, \\ Messina; ${ }^{2}$ Department of Biomolecular Strategies, Genetics, Vanguard Therapies and Neuroscience, I.E.ME.S.T., \\ Palermo; ${ }^{3}$ Department of Chemical, Biological, Pharmaceutical and Environmental Sciences, University of Messina, \\ Messina, Italy
}

\begin{abstract}
Cerebral Cavernous Malformations (CCM) are vascular lesions affecting brain microvessels. While molecular bases of the sporadic condition are not yet well elucidated, familial forms arise following mutations at three different loci KRIT1, CCM2 and $P D C D 10$. However, no germline mutations are detected in a small percentage of families with hereditary history of CCM. In order to detect other possible candidate genes, we performed molecular analysis of SERPINII gene in a cohort of patients carrying no mutations in the three CCM loci, aiming to detect mutations likely associated to lesion development. Therefore, we performed molecular analysis of the SERPINII gene in a cohort of 18 unrelated patients affected by both familial and sporadic CCM show-
\end{abstract}

Correspondence: Luigi Donato, Department of Biomedical and Dental Science and of Morphological and Functional Images, University of Messina, Via Consolare Valeria 1, 98125 Messina, Italy.

Tel.: +390902213136.

E-mail:1donato@unime.it

Key words: PDCD10; SERPINI1; CCM pathogenesis; molecular analysis.

Contributions: CS: manuscript writing; LD and SA: molecular analysis execution; RD: study conceptualization; AS: group coordination.

Conflict of interest: The authors have no conflict of interest to declare.

Ethics approval and consent to participate: The study was approved by the local Ethical Committee (A.O.U. "G. Martino") and informed consent was obtained for all the subjects involved in the study.

Received for publication: 3 May 2021

Revision received: 21 July 2021.

Accepted for publication: 21 August 2021.

${ }^{\circ}$ Copyright: the Author(s), 2021

Licensee PAGEPress, Italy

Journal of Biological Research 2021; 94:9838

doi:10.4081/jbr.2021.9838

This article is distributed under the terms of the Creative Commons Attribution Noncommercial License (by-nc 4.0) which permits any noncommercial use, distribution, and reproduction in any medium, provided the original author(s) and source are credited. ing no germline causative mutations. Mutational analysis resulted negative and only few single nucleotide polymorphisms were detected. However, the rs 11284733 SNP was detected in a high percentage of patients affected by familial form of the disease. This SNP occurs within a noncoding exon retained in an alternative spliced SERPINI1 transcript, suggesting its possible role in gene expression regulation.

\section{Introduction}

Intracranial blood vessel disorders include a very heterogeneous group of pathologies both acquired and genetic. Among these, cerebral cavernous malformation (CCM, OMIM \#116860) is the most frequent, reaching its worldwide incidence up to $0.8 \%{ }^{1}$ Involved vessels appear enlarged and tangled due to absence of pericytes and impairment of endothelial cell junctions. Also cell adhesion to the extracellular matrix is lost. These features result in blood-brain barrier dysfunction and in its increased permeability, with consequent gain of bleeding risk. ${ }^{2}$ Together with intracerebral haemorrhage, also seizures, headache, vertigo and focal neurological deficits can represent the main clinical manifestations of the disease. However, only about $70 \%$ of patients manifests symptomatology. ${ }^{3} \mathrm{CCM}$ can arise sporadically or be inherited as autosomal dominant condition. While molecular bases that lead to the sporadic disease are not yet completely clarified, hereditary familial forms are known to be linked to germline mutations at the three loci KRIT1/CCM1 (HGNC:1573; 7q11.221), CCM2/MGC4607 (HGNC:21708; 7p13) and PDCD10/CCM3 (HGNC:8761; 3q26.1). Affected patients develop multiple lesions usually already at childhood. However, they can remain asymptomatic due to variable expressivity of the disease. ${ }^{4}$ Moreover, incomplete penetrance can determine the absence of lesions in mutation carriers. Penetrance was estimated for the three loci to range around $88 \%, 70 \%$ and $66 \%$ for KRIT1, $C C M 2$ and $P D C D 10$, respectively. ${ }^{5}$ Moreover, mutations at the $P D C D 10$ locus result in a more aggressive clinical phenotype characterized by more frequent haemorrhages, if compared with KRIT1 or CCM 2 - linked forms. ${ }^{6}$ The three CCM genes encode for proteins that are involved in angiogenic-related pathways, contributing to maintenance of cell-cell junctions and cell-extra cellular matrix adhesion, ${ }^{7,8}$ to the regulation of apoptosis/proliferation switch of endothelial cells ${ }^{9}$ and to oxidative damage response. ${ }^{2}$ As known, impairment of system defence against Reactive Oxygen Species (ROS) predisposes to cerebrovascular malformation 
onset $^{10}$ and several genetic polymorphisms in the GLO1 and PON1 were associated to an increased risk of CCM development. ${ }^{11}$ To date, more than 300 causative mutations in the CCM genes were reported and the datasets are continuously growing. Moreover, Single Nucleotide Polymorphisms (SNPs) in KRIT1 and CCM2 were linked to different prognosis. ${ }^{12}$ Mutation rate is about $60 \%$, $20 \%$ and $15 \%$ for the three loci respectively, while no germline mutations are detected in about 5\% of patients with familial CCM. ${ }^{13}$ Despite the hypothesis of a fourth CCM gene involved in lesion development is commonly accepted, no other associated loci have to date been detected. Linkage studies published by Liquori et al. revealed a lower frequency of PDCD10 mutations than expected. ${ }^{14}$ This observation has allowed to hypothesize involvement, in CCM pathogenesis, of another gene mapping in the same chromosomic region of $P D C D 10$. Further analyses showed that SERPINI1 gene (HGNC:8943; 3q26.1) is highly close to PDCD10 and the two genes share a common bidirectional promoter. ${ }^{15}$ This structural model is peculiar of homologous gene-pairs. However, no functional correlations are reported for the PDCD10-SERPINI1 gene-pair and their expression profiles are not comparable. SERPINII encodes for neuroserpin, a serine protease inhibitor that regulates tissue-type plasminogen activator (t-PA). ${ }^{16}$ It is organized in cluster with its homologous SERPINI2 (HGNC:8945). The peculiarity of this chromosomal region is that $P D C D 10$ is located within this cluster, together with an another gene, WDR49 (HGNC:26587; Figure 1). Interestingly, extracellular matrix remodelling due to unconventional protease activity of coagulation factors resulted enhanced in CCM-derived endothelial cells. ${ }^{17}$ Based on these observations, we chose to study the SERPIN1 coding sequence in our patients in order to can consider it in CCM pathogenesis.

\section{Materials and Methods}

\section{Cohort selection}

SERPINI1 gene consists of 10 exons and its molecular analysis was conducted on a cohort of 18 Caucasian non-consanguineous CCM patients. Of these, 9 belonged to families in which CCM segregated, while the other 9 had not affected relatives and they were classified as sporadic. Diagnosis of CCM was based on anamnesis information and magnetic resonance imaging. The previous mutational analysis performed on CCM genes revealed no germline mutations, as well as Multiplex Ligation-dependent Probe Amplification analysis highlighted lo large genomic rearrangements.

\section{Molecular analysis}

DNA was purified from peripheral blood and SERPINI1 coding, noncoding exons and intron-exons boundaries were amplified by polymerase chain reaction and sequenced on 3500 Genetic Analyzer (Thermo Fisher Scientific) by the BigDye Terminator v3.1 chemistry (Applied Biosystems), following manufacturer's guidelines. Primer sequences and reaction conditions are available upon request. The effects of detected variants were in-silico predicted by the SIFT dbSNP, ${ }^{18}$ MutationTaster ${ }^{19}$ and PolyPhen-2 ${ }^{20}$ tools.

The study was approved by the local Ethical Committee (A.O.U. "G. Martino") and informed consent was obtained for all the subjects involved in the study.

\section{Results}

Both coding and noncoding exons of SERPINI1 gene were sequenced but no mutations in the patients were detected. However, four different SNPs were identified and they are listed in Table 1. Their distribution is not equal between sporadic and famil-ial patients. In detail, three SNPs were detected in the sporadic cohort and these are the rs33917740 and the rs34582040, carried by the 3 same patients and the rs2229697, identified in only anoth-er sample. In contrast, the rs 11284733 was detected in 6 patients affected by familial CCM. About their functional consequences, the rs33917740 c.21C $>\mathrm{G}$ (Figure 2a) results in the p.Phe7Leu amino acid substitution in the neuroserpin protein. However, SIFT dbSNP, MutationTaster and PolyPhen-2 tools predicted that it is well tolerated and notdisrupting for protein structure. The rs34582040 c.51A $>\mathrm{G}$ and the rs2229697 c.576G $>$ C are both syn-onymous substitutions, p.Thr17 $=$ and p.Ser192=, respectively. Finally, the rs11284733 c.980-22delAA (Figure 2b) is a dinu-cleotide deletion occurring in a non-coding exon.

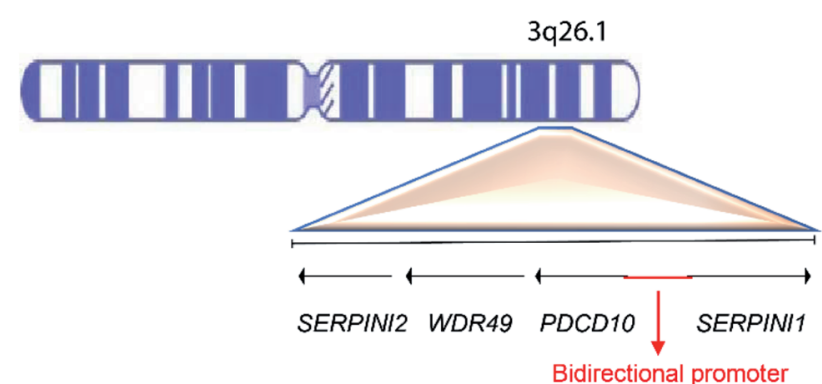

Figure 1. SERPINI1-SERPINI2 gene cluster. The genomic organization of the $3 \mathrm{q} 26$ region shows as PDCD10 and WDR49 genes are included within the SERPINI cluster. The arrows indicate the direction of gene transcription (SERPINI2, WDR49 and PDCD10: reverse strand; SERPINI1: forward strand). The 851 bp SERPINI1$P D C D 10$ bidirectional promoter is highlighted in red.

Table 1. SNP report. Single nucleotide polymorphisms (SNPs) detected in both familial and sporadic CCM patients are listed. For each SNP, the nucleotide and amino acid substitution, frequency in patient cohort and Minor Allele Frequency (MAF) according to the GnomAD Database (https://gnomad.broadinstitute.org/) are reported.

\begin{tabular}{lcccc} 
Variant & Variant effect & $\begin{array}{c}\text { SNP frequency (\%) } \\
\text { in familial cases }\end{array}$ & $\begin{array}{c}\text { SNP frequency (\%) } \\
\text { in sporadic cases }\end{array}$ & $\begin{array}{c}\text { MAF } \\
\text { (GnomAD database) }\end{array}$ \\
rs33917740 & c.21C>G; p.Phe7Leu & 0 & 33 & 0.1262 \\
rs34582040 & c.51A $>$ G; p.Thr17= & 0 & 33 & 0.1030 \\
\hline rs2229697 & c.576G $>$ C; p.Ser192 & 0 & 11 & 0.1263 \\
rs11284733 & c.980-22delAA & 67 & 0 & 0.00007422 \\
\hline
\end{tabular}




\section{Discussion}

The study aimed to evaluate SERPINI1 gene mutations as possible cause of CCM development. SERPINI1 encodes for the neuroserpin, a serin-protease that acts by inhibiting t-PA. It is highly expressed in fetal brain where guides axonal growth and synaptic plasticity. In adults, its expression is limited to few cerebral areas, including hippocampus, amygdala and hypothalamus. If mutated, it causes a neurodegenerative disease known as FENIB (Familial Encephalopathy with Neuroserpin Inclusion Bodies). ${ }^{21}$ According to the observation that SERPINI1 shares its promoter with $P D C D 10$, the third CCM causative gene, we wanted to investigate about its possible role in CCM onset and development. Molecular analysis of SERPINI1 gene in a cohort of patients affected by both familial and sporadic CCM and with no CCM genes mutations allowed us to detect two highly represented SNPs. The rs33917740 is a nucleotide substitution c. $21 \mathrm{C}>\mathrm{G}$ that leads to the p.Phe7Leu amino acid change. Its frequency is homogeneous among the different ethnic groups and ranges between 0.05 and 0.2 worldwide (https:/gnomad.broadinstitute.org/variant/3-167789149-CG?dataset=gnomad_r3). The rs11284733 (c.980-22delAA) is a deletion that occurs in a non-coding exon. This exon results retained in an alternative spliced SERPINII transcript (Ensembl transcript ID: ENST00000494666). The rs11284733 (c.98022delAA) allele was detected only in patients affected by familial forms with a frequency equal to 0.335 . However, its worldwide frequency is estimated to be 0.00007422 , as reported in GnomAD database (https://gnomad.broadinstitute.org/variant/3-167822953-
GAA-G?dataset=gnomad_r3). Therefore we think that this difference is deserving of further investigations. In particular, the role of the SERPINI1 ENST00000494666 transcript could be considered in regulation of CCM gene expression, being it reported in the ANGIOGENES database (http://angiogenes.uni-frankfurt.de/transcript/ENST00000494666), a database collecting both coding and noncoding genes involved in angiogenesis. So, a possible regulatory mechanism for the three CCM genes is not to be excluded.

Moreover, association data do not report any linkage between the rs 11284733 and the FENIB phenotype. FENIB is a rare dominant condition linked to SERPINI1 mutations, characterized by dementia, seizure and progressive myoclonic epilepsy, as consequence of precipitation in neurons of mutated neuroserpin. ${ }^{22}$ In order to describe the possible involvement of SERPINI1 also in CCM development, we previously characterized two different SNPs affecting PDCD10/SERPINII bidirectional promoter and a reduced expression level was observed for $P D C D 10$. However, they seem not to affect SERPINII expression. ${ }^{23,24}$ Neuroserpin acts by inactivating t-PA. In CNS, tPA cleaves both Matrix Metallopeptidase 2 (MMP-2) and MMP-9 enhancing their activity and extra-cellular matrix remodelling rate. ${ }^{25}$ Role of serine proteases and their inhibitors is becoming clearer and it was shown that they are required for brain vasculature development in mice. ${ }^{26}$ Likewise, serine proteases enhance pericyte coverage on the endothelial tubes under pathological conditions. ${ }^{27}$

This study represents the first investigation about role of the tPA inhibitor neuroserpin in CCM development and it was driven by the observation that SERPINI1 gene shares its promoter with $P D C D 10$, the third CCM causative gene. Although no mutations

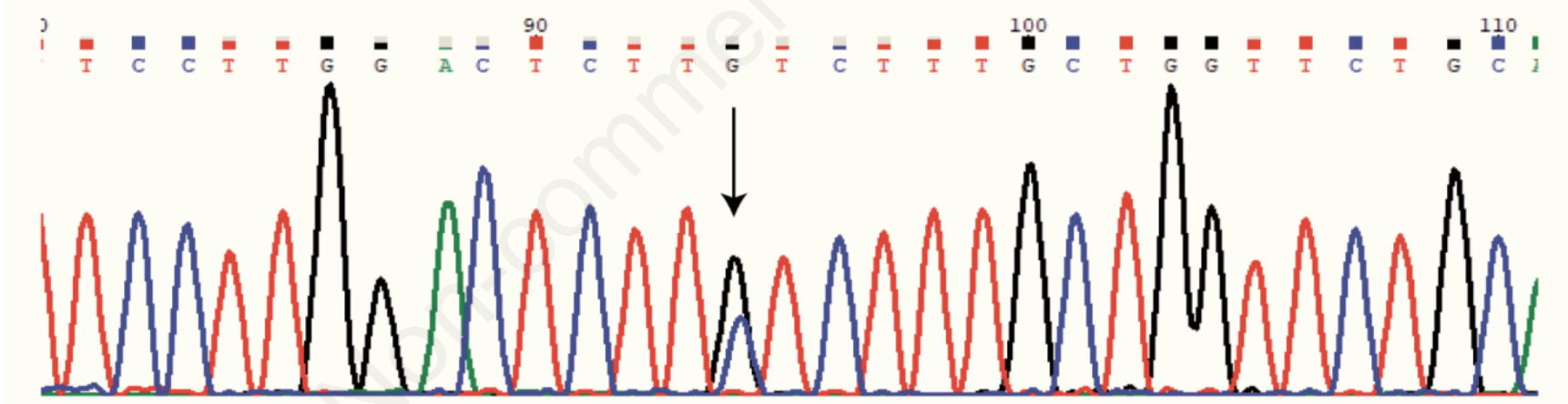

a)

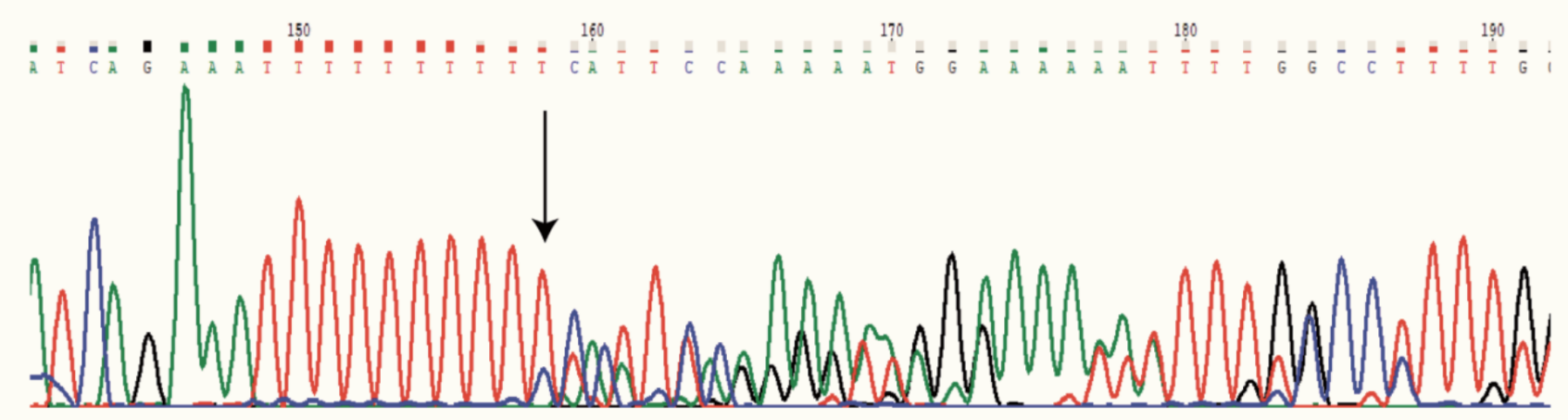

b)

Figure 2. SERPINI1 variants. The electropherograms show the two SERPINI1 SNPs main represented in our cohort of CCM patients. a) The rs33917740 c.21C $>$ G leading to the missense p.Phe7Leu variant. b) The rs11284733 resulting in the c.980-22del intronic deletion. The arrows indicate the mutated nucleotide. 
were found in our cohort of patients, the high frequency of the rs 11284733 in patients affected by the familial form of the disease encourages deepening on the research. Clearly, the reduced sample size makes results provisional requiring a larger scale screening and functional validation of collected data.

\section{Conclusions}

This study describes results obtained by sequencing analysis of SERPINI1 gene performed on a cohort of CCM patients and no mutations were detected. However, we identified the rs11284733 in a high percentage of patients affected by familial CCM. By comparison of allele frequency, this SNP resulted more widely represented in our cohort than in general population. Therefore, although involvement of SERPINII in CCM pathogenesis is to date not confirmed, we think that the regulatory role of the noncoding SERPINII ENST00000494666 transcript in angiogenesis and CCM development requires further investigations.

\section{References}

1. Flemming KD. Incidence, prevalence, and clinical presentation of Cerebral Cavernous Malformations. Methods Mol Biol 2020;2152:27-33.

2. Wei S, Li Y, Polster SP, et al. Cerebral Cavernous Malformation proteins in barrier maintenance and regulation. Int J Mol Sci 2020;21:675.

3. Vercelli GG, Cofano F, Santonio FV, et al. Natural history, clinical, and surgical management of Cavernous Malformations. Methods Mol Biol 2020;2152:35-46.

4. Scimone C, Bramanti P, Ruggeri A, et al. Detection of novel mutation in CCM3 causes familial Cerebral Cavernous Malformations. J Mol Neurosci 2015;57:400-3.

5. Scimone C, Donato L, Katsarou Z, et al. Two novel KRIT1 and CCM2 mutations in patients affected by Cerebral Cavernous Malformations: new information on CCM2 penetrance. Front Neurol 2018;9:953.

6. Wang K, Zhou HJ, Wang M. CCM3 and Cerebral Cavernous Malformation disease. Stroke Vasc Neurol 2019;4:67-70.

7. Johnson AM, Roach JP, Hu A, et al. Connexin 43 gap junctions contribute to brain endothelial barrier hyperpermeability in familial Cerebral Cavernous Malformations type III by modulating tight junction structure. FASEB J 2018;32:2615-29.

8. Dejana E, Orsenigo F. Endothelial adherens junctions at a glance. J Cell Sci 2013;126:2545-49.

9. Zhu Y, Wu Q, Fass M, et al. In vitro characterization of the angiogenic phenotype and genotype of the endothelia derived from sporadic Cerebral Cavernous Malformations. Neurosurgery 2011;69:722-31.

10. Amelina IP, Solovieva EY. Oxidative stress and inflammation as links in a chain in patients with chronic cerebrovascular diseases. Zh Nevrol Psikhiatr Im S S Korsakova 2019;119:106-14.

11. Rinaldi C, Bramanti P, Famà A, et al. Glyoxalase I A111E, paraoxonase I Q192R and L55M polymorphisms in Italian patients with sporadic Cerebral Cavernous Malformations: a pilot study. J Biol Regul Homeost Agents 2015;29:493-500.

12. Rinaldi C, Bramanti P, Scimone C, et al. Relevance of CCM gene polymorphisms for clinical management of sporadic Cerebral Cavernous Malformations. J Neurol Sci 2017;380:31-7.

13. Riolo G, Ricci C, Battistini S. Molecular genetic features of Cerebral Cavernous Malformations (CCM) patients: an overall view from genes to endothelial cells. Cells 2021;10:704.

14. Liquori CL, Berg MJ, Squitieri F, et al. Low frequency of PDCD10 mutations in a panel of CCM3 probands: potential for a fourth CCM locus. Hum Mutat 2006;27:118.

15. Chen PY, Chang WS, Chou RH, et al. Two non-homologous brain diseases-related genes, SERPINI1 and PDCD10, are tightly linked by an asymmetric bidirectional promoter in an evolutionarily conserved manner. BMC Mol Biol 2007;8:2.

16. Silverman GA, Bird PI, Carrell RW, et al. The serpins are an expanding superfamily of structurally similar but functionally diverse proteins. Evolution, mechanism of inhibition, novel functions, and a revised nomenclature. J Biol Chem 2001;276:33293-6.

17. Scimone C, Donato L, Alibrandi S, et al. Transcriptome analysis provides new molecular signatures in sporadic Cerebral Cavernous Malformation endothelial cells. Biochim Biophys Acta Mol Basis Dis 2020;1866:165956.

18. Vaser R, Adusumalli S, Leng SN,et al. SIFT missense predictions for genomes. Nat Protoc. 2016 Jan;11(1):1-9.

19. Steinhaus R, Proft S, Schuelke M, et al. MutationTaster2021. Nucleic Acids Res. 2021 Jul 2;49(W1):W446-W451.

20. Adzhubei IA, Schmidt S, Peshkin L, et al. A method and server for predicting damaging missense mutations. Nat Methods. 2010 Apr;7(4):248-9.

21. Davis RL, Shrimpton AE, Carrell RW, et al. Association between conformational mutations in neuroserpin and onset and severity of dementia. Lancet 2002;359:2242-7.

22. Ali MF, Kaushik A, Gupta D, et al. Changes in strand $6 \mathrm{~B}$ and helix B during neuroserpin inhibition: Implication in severity of clinical phenotype. Biochim Biophys Acta Proteins Proteom 2020;1868:140363.

23. Scimone C, Bramanti P, Ruggeri A, et al. CCM3/SERPINI1 bidirectional promoter variants in patients with Cerebral Cavernous Malformations: a molecular and functional study. BMC Med Genet 2016;17:74.

24. Scimone C. Possible related functions of the non-homologous co-regulated gene pair PDCD10 and SERPINI1. EMBJ 2017;12:041-6.

25. Omouendze PL, Henry VJ, Porte B, et al. Hypoxia-ischemia or excitotoxin-induced tissue plasminogen activator- dependent gelatinase activation in mice neonate brain microvessels. PLoS One 2013;8:e71263.

26. Maroney SA, Westrick RJ, Cleuren AC, et al. Tissue factor pathway inhibitor is required for cerebrovascular development in mice. Blood 2021;137:258-68.

27. Hu E, Hu W, Yang A, et al. Thrombin promotes pericyte coverage by Tie 2 activation in a rat model of intracerebral hemorrhage. Brain Res 2019;1708:58-68. 